\title{
Improved Attentional Performance Following Citicoline Administration in Healthy Adult Women
}

\author{
Erin McGlade ${ }^{1,2}$, Allison Locatelli ${ }^{1}$, Julia Hardy ${ }^{1}$, Toshikazu Kamiya ${ }^{3}$, Masahiko Morita ${ }^{4}$, \\ Koji Morishita ${ }^{4}$, Yoichiro Sugimura ${ }^{4}$, Deborah Yurgelun-Todd ${ }^{1,2}$ \\ ${ }^{1}$ Brain Institute, University of Utah, Salt Lake City, USA; ${ }^{2}$ Department of Psychiatry, University of Utah, Salt Lake City, USA; \\ ${ }^{3}$ Kyowa Hakko USA, Inc., New York, USA; ${ }^{4}$ Kyowa Hakko Bio Co., Ltd., Tokyo, Japan. \\ Email: erin.mcglade@hsc.utah.edu
}

Received February 23 ${ }^{\text {rd }}, 2012$; revises April 16 ${ }^{\text {th }}, 2012$; accepted April 23 ${ }^{\text {rd }}, 2012$

\begin{abstract}
Objectives: The present study assessed the potential cognitive-enhancing effects of citicoline, a dietary supplement, in healthy adult women. Specifically, it was hypothesized that citicoline supplementation would be associated with improved attention compared to placebo. Methods: The investigation was a double-blind, randomized, placebo-controlled three-arm study. Sixty healthy adult women ages 40 - 60 completed a clinical screening visit, including a medical exam. After study enrollment each subject was randomly assigned to one of three groups: a daily oral dose of $250 \mathrm{mg}$ citicoline, $500 \mathrm{mg}$ citicoline, or placebo for 28 days. Participants were evaluated with the Continuous Performance Test II (CPT-II), a measure sensitive to attentional function, during a baseline visit and 28 days after baseline. Results: All 60 participants were included in the analyses, which included an ANOVA with Tukey's post-hoc tests and $t$-tests. After 28 days of supplementation, individuals in the $250 \mathrm{mg}$ group made fewer omission $(p=0.04)$ and commission $(p=0.03)$ errors compared to those in the placebo group. Individuals in the $500 \mathrm{mg}$ group made significantly fewer commission errors compared to those in the placebo group $(p=0.03)$ and trended toward making fewer omission errors $(p=0.07)$. Conclusion: After 28 days of daily citicoline supplementation, participants who were administered either the $250 \mathrm{mg}$ or the $500 \mathrm{mg}$ citicoline doses showed significantly better ability to produce correct responses on the CPT-II, likely due to improved cognitive inhibition. Our findings suggest that citicoline may improve attentional performance in middle-aged women and may ameliorate attentional deficits associated with central nervous system disorders.
\end{abstract}

Keywords: Citicoline; Continuous Performance Test; Attention

\section{Introduction}

Citicoline is a natural product that is marketed as a nutritional supplement in the United States. Exogenous administration of citicoline (CDP-choline; cytidine 5'-diphosphocholine) has been shown to influence brain cellular metabolism and citicoline has demonstrated a variety of cognitive-enhancing and neuroprotective properties in pre-clinical and clinical studies [1-3]. A compound composed of choline and cytidine, citicoline is instrumental in the synthesis of cell membrane phospholipids that aid in neuronal membrane repair [3,4], and provides a choline source needed for acetylcholine (ACh) and phosphatidylcholine synthesis [3,5].

Pharmacokinetic studies on the absorption of citicoline through the oral route have shown that the drug is rapidly absorbed with less than one percent excreted in the feces. Citicoline is metabolized during intestinal absorption yielding choline and uridine [6]. After metabolism, citicoline is distributed throughout the body by way of sys- temic circulation in the form of choline and uridine for utilization in many biosynthetic pathways. After crossing the blood-brain barrier, uridine is synthesized into uridine 5'-triphosphate, which can be further metabolized to cytidine triphosphate and CDP-choline [6].

Previous studies on supplementation with citicoline have shown an increase in choline-containing compounds associated with improved bioenergetics. Specifically, Babb and colleagues applied proton magnetic resonance spectroscopy (MRS) to measure in-vivo brain chemistry and found that a single oral dose of CDP-choline resulted in increased plasma choline in older (mean age 59 years) and younger (mean age 25) participants three hours after supplementation [7]. These investigators subsequently [8] applied phosphorous MRS to measure changes in brain chemistry in healthy older adults following 6 weeks of $500 \mathrm{mg}$ citicoline supplementation. The study findings showed an increase in brain phosphodiesters that correlated with memory improvement as measured by the 
California Verbal Learning Test [8]. To further examine the potential effects of citicoline supplementation on brain metabolism, Silveri and colleagues used phosphorrus MRS [9] to measure brain metabolites in healthy adults 40 - 60 years old who received citicoline supplementation for six weeks at doses of either 500 or 2000 $\mathrm{mg} /$ day. Increased phosphocreatine levels were observed in the anterior cingulate, suggesting improved bioenergetics and enhanced phospholipid membrane maintenance in the frontal lobe. Animal study results have complemented these data on human brain changes by indicating a potential role of citicoline in the moderation of neurotransmitters. In a study involving exogenous citicoline administration to rats for 28 days, supplementation increased dopamine release in the striatum [10].

A number of investigations have focused on the therapeutic effects of citicoline in patients who have suffered from stroke or a traumatic brain injury. Patients with neurocognitive decline following an initial stroke have shown improvement in both attention and executive functioning after six months of $1000 \mathrm{mg} /$ day citicoline treatment $[4,11,12]$. In addition, Agnoli and colleagues [12] showed that citicoline supplementation in an elderly sample of individuals with mild to moderate memory deficits resulted in significantly improved memory and attention. They found that individuals who took $1000 \mathrm{mg}$ of citicoline for six weeks showed improved acquisition efficiency and global memory efficiency, as measured by the Randt Memory Test, compared to individuals who took the placebo. The authors interpreted these findings as indicative of memory processing and attentional improvement associated with dopaminergic stimulation from citicoline consumption [12].

While previous studies of citicoline have focused on study populations with neurological conditions or significant cognitive deficits, there is a paucity of data on the effects of citicoline in normal aging. Aging in the absence of acute or chronic medical conditions has been associated with decreased attentional capacity and increased distractibility [13,14]. Research examining brain structure and function as well as cognitive performance has shown a number of changes in healthy aging adults. For example, Madden and colleagues [15] found that older and younger adults showed similar prefrontal activation during a visual target response task; however, older adults also showed increased deep gray matter activation. Regression analyses suggested that younger adults' performance on the visual target response task was mediated by the prefrontal cortex, whereas deep gray matter structures were more involved in older adults' task performance. In addition, older adults showed slower response times compared to the younger adults. Vaidya and colleagues [16] applied brain imaging methods and showed smaller rostral ACC gray matter volume and decreased blood flow in dorsal and rostral ACC regions associated with age. These findings are consitent with other neuroimaging studies that have reported reduced brain activity and decreased cerebral glucose metabolism, as well as reduced performance on response inhibition tasks with age [17-20]. The brain changes observed during aging in healthy adults suggest a potential role for citicoline supplementation.

The current study is a double-blind, placebo-controlled, three-arm study designed to evaluate the effect of two doses of citicoline on attention after four weeks of supplementation. Attentional performance measures in healthy, aging adult women were monitored in a trial involving two different doses of oral citicoline $(250 \mathrm{mg} / \mathrm{day}$ and $500 \mathrm{mg} /$ day) or a placebo. The study population was limited to women to decrease heterogeneity given hormonal changes that males and females experience between 40 and 60 years old. It was hypothesized that the women who received citicoline would demonstrate improved attentional performance on a computerized attention task compared to the women who received the placebo.

\section{Subjects and Methods}

\subsection{Subjects}

Sixty healthy female adults ranging from 40 to 60 years of age $(M=47.38, S D=5.48)$ were recruited from Salt Lake City, UT (Table 1). The participants were screened prior to enrollment to ensure that they met the inclusion criteria for the study. Potential participants were enrolled if they had no significant medical conditions, no history of psychiatric disorders, no current Axis I or II diagnosis, and no history of previous participation in a pharmacotherapeutic trial. The study was conducted under the guidelines of the University of Utah Institutional Review Board. All participants provided written informed consent per the IRB and Declaration of Helsinki, and participants were compensated financially for their time.

\subsection{Study Design}

All participants who were screened positive for study

Table 1. Baseline subject demographics.

\begin{tabular}{|c|c|c|c|}
\hline & $\begin{array}{l}250 \mathrm{mg} \\
(\mathrm{N}=20)\end{array}$ & $\begin{array}{l}500 \mathrm{mg} \\
(\mathrm{N}=20)\end{array}$ & $\begin{array}{l}\text { Placebo } \\
(N=20)\end{array}$ \\
\hline Age \pm SD & $46.00 \pm 4.60$ & $48.20 \pm 5.99$ & $47.95 \pm 5.74$ \\
\hline $\begin{array}{c}\text { Education } \pm \text { SD } \\
\text { (years) }\end{array}$ & $16.53 \pm 2.37$ & $17.75 \pm 1.92$ & $16.30 \pm 2.54$ \\
\hline Height (in) \pm SD & $64.22 \pm 3.59$ & $63.93 \pm 2.28$ & $64.30 \pm 3.18$ \\
\hline Weight (lbs) \pm SD & $158.38 \pm 26.67$ & $147.25 \pm 40.15$ & $154.80 \pm 34.74$ \\
\hline $\begin{array}{l}\text { Vocabulary } \\
\text { Score } \pm \text { SD }\end{array}$ & $105.15 \pm 17.64$ & $109.75 \pm 11.48$ & $103.60 \pm 11.55$ \\
\hline
\end{tabular}


entry then completed a clinical screening visit, including a medical exam. Participants also completed a diagnostic interview, lifestyle questionnaires, vocabulary subtest of the Wechsler Adult Intelligence Scale-Fourth Edition (WAIS-IV), California Verbal Learning Test (CVLT), and the Continuous Performance Test II (CPT-II) during their initial visit. Following the completion of this first visit, subjects were randomly assigned to a dosing group of $250 \mathrm{mg}, 500 \mathrm{mg}$, or placebo and given their 28-day supply of oral citicoline (Cognizin ${ }^{\circledR}$ Kyowa Hakko Kogyo Co., Ltd., Japan) or placebo. Both participants and researchers remained blind to the treatment condition for the duration of data collection. Participants were instructed to take one capsule per day every day for the next 28 days. Participants returned on day 28 to repeat assessment measures, including the CPT-II and the medical exam.

The CPT-II is a test of sustained attention that has been adapted for computerized administration. It has been shown to be a reliable measure for sustained and selective attention [21,22]. The CPT-II was completed on an IBM-compatible laptop computer. The task begins with a short practice exercise then proceeds into the full test, which lasts for 14 minutes. For both the practice test and the full test, participants are instructed to press the spacebar as quickly and as accurately as possible when any letter other than $\mathrm{X}$ appears on the screen. Letters appear on the computer screen 1 letter at a time for 250 milliseconds. Inter-stimulus intervals between letter displays vary between 1,2 , and 4 seconds. Measures that are provided by the CPT-II assessment include omission and commission errors. Omission errors occur when the participant does not respond when they should respond (i.e., not pressing the spacebar when a letter other than $\mathrm{X}$ appears), and commission errors occur when the participant fails to inhibit a response (i.e., pressing the spacebar when the letter $X$ appears). Computerized scoring is completed automatically after the administration of the CPT-II [23]. The CPT-II was administered at baseline and at day 28, which was the last day of oral supplementation.

\section{Results}

Sixty healthy adult women participated in the study. Three additional women enrolled in the study but were discontinued due to study-unrelated events. The sixty women were divided into three groups of 20. One group took the low dose of citicoline (250 mg), one group took the high dose of citicoline $(500 \mathrm{mg})$, and one group took a placebo for 28 days.

Between-group analyses prior to supplementation showed no group difference on estimated intelligence as measured by WAIS-IV Vocabulary $(p=0.35)$. Mean estimated IQs for each group fell within the average range $(250 \mathrm{mg}=105.15,500 \mathrm{mg}=109.75$, placebo $=$ 103.60). Baseline measures of verbal memory, as assessed by CVLT, also indicated no between-group differences in performance ( $p=0.23$ on CVLT Lists $1-5$, indicating similar memory before supplementation).

Additionally, analyses of CPT-II response results from baseline and day 28 were performed with SPSS (PASW Statistics 18.0). All $p$-values are reported as one-tailed because a priori hypotheses specified improvement on CPT-II scores after supplementation. Additionally, one woman in the $500 \mathrm{mg}$ group was excluded from CPT-II analyses due to the fact that her attention performance score at visit 3 was a clear outlier (49 standard deviations above the group mean).

\section{Comparisons between Groups for Day 28 Scores}

An ANOVA and $t$-tests were completed to assess between group differences at visit 3 . Analysis of attentional performance at visit 3 that included all three groups showed overall significant between group differences on commission $(p=0.02)$ and omission errors $(p=0.03)$. Tukey's post-hoc tests showed that individuals in the 250 mg group performed fewer omission $(p=0.04)$ and commission $(p=0.03)$ errors compared to those in the placebo group. Individuals in the $500 \mathrm{mg}$ group also made significantly fewer commission errors compared to those in the placebo group $(p=0.03)$ and trended toward significance making fewer omission errors $(p=0.07)$ (Tables 2 and 3).

$T$-tests were also completed to compare the $250 \mathrm{mg}$ group to the placebo group and the $500 \mathrm{mg}$ group to the placebo group individually. Similar to the results of the ANOVA, individuals in the $250 \mathrm{mg}$ group made fewer omission $(p=0.04)$ and commission $(p=0.02)$ errors compared to those in the placebo group. Individuals in the $500 \mathrm{mg}$ group made fewer commission errors compared to those in the placebo group $(p=0.02)$ and trended toward making fewer omission errors $(p=0.06)$.

\section{Discussion}

Our study findings indicate that citicoline supplementation was associated with improved attentional focus and inhibition, as measured by the CPT-II, in a group of healthy female adult volunteers. Participants in the three groups showed no differences in age, years of education, height, weight, or estimated levels of global cognitive functioning at baseline. After 28 days both supplementation groups (250 $\mathrm{mg}$ and $500 \mathrm{mg}$ ) evidenced improved inhibition. Additionally, the $250 \mathrm{mg}$ group evidenced improved attentional focus at time 3 , while the $500 \mathrm{mg}$ group trended toward showing improved focus. These results indicate that citicoline enhanced two aspects of 
Table 2. $250 \mathrm{mg}$ compared to placebo: CPT-II omission and commission errors at time 3 .

\begin{tabular}{ccc}
\hline & $\mathbf{2 5 0} \mathbf{~ m g ~ ( N = ~ 2 0 )}$ & Placebo $\mathbf{( N = \mathbf { 2 0 } )}$ \\
\hline $\begin{array}{c}\text { Time 3 CPT-II Omission } \\
\text { Errors Mean } \pm \text { SE }\end{array}$ & $0.90 \pm 0.25$ & $6.70 \pm 3.11$ \\
$\begin{array}{c}\text { Time 3 CPT-II Commission } \\
\text { Errors Mean } \pm \text { SE }\end{array}$ & $2.70 \pm 0.77$ & $5.50 \pm 1.03$ \\
\hline
\end{tabular}

Table $3.500 \mathrm{mg}$ compared to placebo: CPT-II omission and commission errors at time 3 .

\begin{tabular}{ccc}
\hline & $\mathbf{5 0 0} \mathbf{~ m g} \mathbf{( N = 1 9 )}$ & Placebo $\mathbf{( N = \mathbf { 2 0 } )}$ \\
\hline $\begin{array}{c}\text { Time 3 CPT-II Omission } \\
\text { Errors Mean } \pm \text { SE }\end{array}$ & $1.47 \pm 0.66$ & $6.70 \pm 3.11$ \\
$\begin{array}{c}\text { Time 3 CPT-II Commission } \\
\text { Errors Mean } \pm \text { SE }\end{array}$ & $2.68 \pm 0.75$ & $5.50 \pm 1.03$ \\
\hline
\end{tabular}

attention-attentional focus and inhibition in healthy women ages 40 - 60 .

Prior research has shown that citicoline administration improves attention in a variety of patient populations, including individuals with neurocognitive degeneration after a stroke [4,11] and elderly participants [12]. However, the population of the current study differs from previous studies, as the current study included only healthy middle-aged women rather than clinical patient populations. Whereas prior researchers have focused on populations with deficits in cognitive performance, including impaired memory or attention, the current study examined women with estimated intelligence and verbal memory within the average range. Findings from this study extend previous research by showing that citicoline administration improves attention not only in clinical populations but also in healthy female adults. Additionally, previous studies have focused on higher doses of citicoline, generally between 1000 and 2000 mg. However, the current study shows improved attention in healthy women between the ages of 40 and 60 who received significantly smaller amounts of citicoline, specifically 250 and $500 \mathrm{mg}$. In addition to extending findings from patient populations to healthy aging women using smaller doses of citicoline, the current study also provides behavioral support for prior studies suggesting improved bioenergetics in the frontal lobe [9], where much of attentional focus is believed to occur.

One mechanism underlying the observed improvement in performance in the current study is likely related to dopamine transmission. As individuals age, dopaminergic transmission and the availability of dopamine receptors decrease, contributing to decreased performance on attention and executive function measures that occurs as individuals age [24,25]. Conversely, dopaminergic uptake has been associated with improved performance on working memory and executive function tasks requiring attention [25,26]. Previous investigations have shown that citicoline increases dopamine [10,27], increases dopamine receptor densities [28], and aids in protection of neurons related to dopamine [29]. While dopamine was not measured in the current study, it seems likely that dopamine increases identified with citicoline supplementation may explain the improved performance on attention tasks shown in the present study.

Given the evidence for improved attention with citicoline supplementation provided by the current study, this study could be extended in multiple ways. The current study includes women between the ages of 40 and 60 , which limits generalizability to a broader population. It is possible that the effects of citicoline supplementation may be different for men given biological differences, especially considering hormonal changes occurring in middle age. However, the effects of citicoline supplementation at lower doses (e.g. 250 and $500 \mathrm{mg}$ ) with healthy men could be examined in future studies. Future research examining the effects of citicoline on a more diverse sample is warranted as well, given the fact that the current sample consisted primarily of Caucasian individuals. Because the participants in this study were healthy, it is unclear to what extent the results of the current study may be used to inform the effects of citicoline supplementation with more ill populations, including individuals with brain injury or psychiatric disorders. Given the significant role of dopamine in a number of psychiatric disorders, and research suggesting that citicoline affects dopaminergic systems, additional research focused on citicoline supplementation with psychiatric populations is warranted.

To our knowledge, the current study is the first to examine the effects of low doses of citicoline on cognitive performance in healthy female adults. Women who received citicoline showed improved attentional performance after 28 days of supplementation. Results of the current study are consistent with prior studies showing improved cognitive performance after citicoline supplementation in individuals with cognitive deficits. Practical applications of this research include the possible use of citicoline supplementation to ameliorate cognitive deficits associated with healthy aging, but also to ameliorate cognitive deficits associated with psychiatric disorders.

\section{REFERENCES}

[1] R. Ozay, et al., "Citicoline Improves Functional Recovery, Promotes Nerve Regeneration, and Reduces Postoperative Scarring after Peripheral Nerve Surgery in Rats,” Surgical Neurology, Vol. 68, No. 6, 2007, pp. 615-622. doi:10.1016/j.surneu.2006.12.054

[2] V. Parisi, et al., "Evidence of the Neuroprotective Role of Citicoline in Glaucoma Patients," Progress in Brain Research, Vol. 173, 2008, pp. 541-554. 
[3] J. J. Secades and J. L. Lorenzo, "Citicoline: Pharmacological and Clinical Review, 2006 Update,” Methods \& Findings in Experimental \& Clinical Pharmacology, Vol. 28, Suppl. B, 2006, pp. 1-56.

[4] R. Conant and A. G. Schauss, "Therapeutic Applications of Citicoline for Stroke and Cognitive Dysfunction in the Elderly: A Review of the Literature," Alternative Medicine Review, Vol. 9, No. 1, 2004, pp. 17-31.

[5] I. H. Ulus, et al., "Choline Increases Acetylcholine Release and Protects against the Stimulation-Induced Decrease in Phosphatide Levels within Membranes of Rat Corpus Striatum,” Brain Research, Vol. 484, No. 1-2, 1989, pp. 217-227.

[6] R. J. Wurtman, et al., "Effect of Oral CDP-Choline on Plasma Choline and Uridine Levels in Humans," Biochemical Pharmacology, Vol. 60, No. 7, 2000, pp. 989992. doi:10.1016/S0006-2952(00)00436-6

[7] S. M. Babb, et al., "Differential Effect of CDP-Choline on Brain Cytosolic Choline Levels in Younger and Older Subjects as Measured by Proton Magnetic Resonance Spectroscopy," Psychopharmacology, Vol. 127, No. 2, 1996, pp. 88-94. doi:10.1007/BF02805979

[8] S. M. Babb, et al., "Chronic Citicoline Increases Phosphodiesters in the Brains of Healthy Older Subjects: An in Vivo Phosphorus Magnetic Resonance Spectroscopy Study," Psychopharmacology, Vol. 161, No. 3, 2002, pp. 248-254. doi:10.1007/s00213-002-1045-y

[9] M. M. Silveri, et al., "Citicoline Enhances Frontal Lobe Bioenergetics as Measured by Phosphorus Magnetic Resonance Spectroscopy,” NMR in Biomedicine, Vol. 21, No. 10, 2008, pp. 1066-1075. doi:10.1002/nbm.1281

[10] J. Agut, J. Ortiz and R. Wurtman, "Cytidine (5')Diphosphocholine Modulates Dopamine K(+)-Evoked Release in Striatum Measured by Microdialysis," Annals of the New York Academy of Sciences, Vol. 920, 2000, pp. 332- 335. doi:10.1111/j.1749-6632.2000.tb06944.x

[11] A. Davalos and J. Secades, "Citicoline Preclinical and Clinical Update 2009-2010,” Stroke, Vol. 42, No. 1, 2011, pp. S36-S39. doi:10.1161/STROKEAHA.110.605568

[12] A. Agnoli, G. Bruno and M. Fioravanti, "Therapeutic Approach to Senile Memory Impairment: A Double-Blind Clinical Trial with CDP Choline,” Alzheimer's Disease: Proceedings of the 5th Meeting of the International Study Group on the Pharmacology of Memory Disorders Associated with Aging, Birkhauser, Boston, 1989.

[13] G. Hochanadel and E. Kaplan, "Neuropsychology of Normal Aging, in Clinical Neurology of Aging,” In: M. Albert, Ed., Oxford University Press, New York, 1984, pp. 231-244.

[14] J. McDowd and J. Birren, “Aging and Attentional Processes, in Handbook of the Psychology of Aging," In: J. Birren and K. Schaie, Eds., Academic Press, San Diego, 1990, pp. 222-233.

[15] D. J. Madden, et al., "Age-Related Changes in Neural Activity during Visual Target Detection Measured by fMRI,” Cerebral Cortex, Vol. 14, 2004, pp. 143-155. doi:10.1093/cercor/bhg113
[16] J. G. Vaidya, et al., “Aging, Grey Matter, and Blood Flow in the Anterior Cingulate Cortex,” Neuroimage, Vol. 37, No. 4, 2007, pp. 1346-1353. doi:10.1016/j.neuroimage.2007.06.015

[17] J. V. Pardo, et al., "Where the Brain Grows Old: Decline in Anterior Cingulate and Medial Prefrontal Function with Normal Aging,” Neuroimage, Vol. 35, No. 3, 2007, pp. 1231-1237. doi:10.1016/j.neuroimage.2006.12.044

[18] M. W. Willis, et al., "Age, Sex and Laterality Effects on Cerebral Glucose Metabolism in Healthy Adults," Psychiatry Research Neuroimaging, Vol. 114, No. 1, 2002, pp. 23-37. doi:10.1016/S0925-4927(01)00126-3

[19] W. L. Whiting, D. J. Madden and K. J. Babcock, “Overriding Age Differences in Attentional Capture with Top-Down Processing," Psychology and Aging, Vol. 22, No. 2, 2007, pp. 223-232.

doi:10.1037/0882-7974.22.2.223

[20] D. H. Spieler, D. A. Balota and M. E. Faust, "Stroop Performance in Healthy Younger and Older Adults and in Individuals with Dementia of Alzheimer's Type,” Journal of Experimental Psychology: Human Perception and Performance, Vol. 22, No. 2, 1996, pp. 461-479. doi:10.1037/0096-1523.22.2.461

[21] C. K. Conners, et al., "Continuous Performance Test Performance in a Normative Epidemiological Sample," Journal of Abnormal Child Psychology, Vol. 31, No. 5, 2003, pp. 555-562. doi:10.1023/A:1025457300409

[22] J. N. Epstein, et al., "Relations between Continuous Performance Test Performance Measures and ADHD Behaviors," Journal of Abnormal Child Psychology, Vol. 31, No. 5, 2003, pp. 543-554. doi:10.1023/A:1025405216339

[23] C. K. Conners, "The Conners Continuous Performance Test,” Multi-Health Systems, Inc., Toronto, 1994.

[24] N. D. Volkow, et al., "Association between Decline in Brain Dopamine Activity with Age and Cognitive and Motor Impairment in Healthy Individuals," American Journal of Psychiatry, Vol. 155, No. 3, 1998, pp. 344349.

[25] A. Nieoullon, "Dopamine and the Regulation of Cognition and Attention,” Progress in Neurobiology, Vol. 67, No. 1, 2002, pp. 53-83. doi:10.1016/S0301-0082(02)00011-4

[26] J. O. Rinne, et al., "Cognitive Impairment and the Brain Dopaminergic System in Parkinson Disease,” Archives of Neurology, Vol. 57, No. 4, 2000, pp. 470-475. doi:10.1001/archneur.57.4.470

[27] R. Rejdak, et al., "Citicoline Treatment Increases Retinal Dopamine Content in Rabbits,” Ophthalmic Research, Vol. 34, No. 3, 2002, pp. 146-149. doi:10.1159/000063658

[28] R. Giménez, J. Raïch and J. Aguilar, "Changes in Brain Striatum Dopamine and Acetylcholine Receptors Induced by Chronic CDP-Choline Treatment of Aging Mice,” British Journal of Pharmacology, Vol. 104, No. 3, 1991, pp. 575-578. doi:10.1080/10623320600934341

[29] K. Radad, et al., "CDP-Choline Reduces Dopaminergic Cell Loss Induced by MPP+ and Glutamate in Primary Mesencephalic Cell Culture," International Journal of Neuroscience, Vol. 117, No. 7, 2007, pp. 985-998. 